\title{
Estudo de prevalência de depressão e síndrome cerebral orgânica na popu- lação de idosos, Brasil
}

\author{
Prevalence of depression and organic cerebral syndrome in the elderly popula- \\ tion (Brazil)
}

\author{
Renato P. Veras*, Evandro da S. F. Coutinho**
}

\begin{abstract}
VERAS, R. P. \& COUTINHO, E. da S.F. Estudo de prevalência de depressão e síndrome cerebral orgânica na população de idosos, Brasil. Rev. Saúde públ., S. Paulo, 25: 209-17, 1991. São apresentados dados de um estudo de prevalência de síndrome cerebral orgânica e depressão em uma população de idosos em três distritos da cidade do Rio de Janeiro, RJ, Brasil, assim como discutem aspectos metodológicos relacionados com a confiabilidade interavaliadores e teste-reteste e estabelecimento do ponto de corte do instrumento utilizado (BOAS). As taxas de prevalência de síndrome cerebral orgânica variaram de $5,9 \%, 9,8 \%$ e $29,8 \%$ entre os distritos estudados, enquanto as taxas de Depressão variaram de $20,9 \%, 23,0 \%$ e $36,8 \%$. Foram ainda calculadas as taxas de prevalência corrigidas pelos dados de sensibilidade e especificidade para ambos os diagnósticos. São discutidos os fatores associados com tais diferenças, tomando como referência a literatura nacional e internacional.
\end{abstract}

Descritores: Idoso. Depressão, epidemiologia. Distúrbios mentais orgânicos, epidemiologia. Métodos epidemiológicos.

\section{Introdução}

Os estudos da epidemiologia da saúde mental no continente latino-americano, vêm, nos últimos anos, despertando maior interesse de pesquisadores, e este fato se traduz pela ampliação da produção na área***. Alguns grupos etários, devido à importante prevalência dos distúrbios mentais e suas características e particularidades, incorporam, cada vez mais, maior número de investigações e interesse acadêmico. Os idosos se enquadram nesta referência, possivelmente pelo reconhecimento de sua importância no campo de intervenção da saúde pública e talvez devido à necessidade de estudos que indiquem com maior acuidade as estimativas de prevalência das doenças mentais e em particular

* Instituto de Medicina Social, da Universidade do Estado do Rio de Janeiro, Rio de Janeiro, RJ - Brasil.

** Instituto de Medicina Social da Universidade do Estado do Rio de Janeiro e Escola Nacional de Saúde Pública-FIOCRUZ - Rio de Janeiro, RJ - Brasil.

*** A lista de autores latino-americanos é extensa; entre os brasileiros, os mais citados são: Almeida Filho e Bastos $^{2}$, Almeida Filho e Santana ${ }^{3}$ Almeida Filho e col ${ }^{5,4}$; Almeida Filho ${ }^{1}$; Mari e Williams ${ }^{26,27,28}$; Ma$\mathrm{ri}^{25}$; Mari e col. ${ }^{29,30}$; Coutinho e col. ${ }^{13}$; Coutinho e Klein ${ }^{14}$; Coutinho ${ }^{12}$; Klein e Coutinho ${ }^{23}$; Morgado e Coutinho ${ }^{33}$; Veras e col. ${ }^{39}$, Miranda e col. ${ }^{32}$; Iacopo$\mathrm{ni}^{19}$; Blay ${ }^{8,9,10}$; Blay e col ${ }^{11}$.

Separatas/Reprints: R.P.Veras - Rua São Francisco Xavier, 524 - 20550 - Rio de Janeiro, RJ - Brasil da Síndrome Cerebral Orgânica e Depressão. Como exemplo, nos estudos selecionados por $\mathrm{Kay}^{21}$ os achados de prevalência para Síndrome Cerebral Orgânica severa variam de $0,6 \%{ }^{20}$ a $5,6 \% .{ }^{22}$ Para demência moderada, o intervalo vai de $5,7 \%{ }^{22}$ a $52,6 \%$. ${ }^{20}$ Deve-se considerar, no entanto, esses estudos não são homogêneos quanto aos instrumentos utilizados, ao tamanho da amostra, às faixas etárias estudadas e aos critérios diagnósticos. Talvez devido à variabilidade dessas taxas, nos Estados Unidos trabalhase com estimativas para demência severa de 5 a $8 \%$ para população acima de 65 anos, e para o grupo etário acima de 80 anos com taxas de 15 a $20 \%{ }^{41}$. Para depressão entre os idosos, o estudo mais citado na literatura, realizado por Gurland e col, ${ }^{27}$ nas cidades de Nova York e Londres, apresentou uma prevalência de $13 \%$, sendo que para a desordem afetiva severa foi de $2,5 \%$. Na Inglaterra, num estudo recente, ${ }^{24}$ a prevalência foi de $13,5 \%$.

Nos últimos anos, a produção na área vem crescendo, e o acúmulo de conhecimento começa a possibilitar uma discussão metodológica inovadora, inclusive quando confrontada com a produzida nos países centrais. Este artigo propõe-se a apresentar algumas hipóteses pouco referidas na literatura. É intenção deste texto discutir a necessidade de adequação do instrumento utilizado na entrevista psiquiátrica, quando do estudo de validação, com o questionário uti- 
lizado pelo projeto e, à luz dos resultados obtidos, apresentar alguns procedimentos que devem ser observados. Também fazem parte do artigo a apresentação do questionário desenvolvido para o estudo domiciliar, o instrumento padronizado para a entrevista psiquiátrica, os resultados e discussão dos estudos de confiabilidade e validação, além da prevalência para Síndrome Cerebral Orgânica e Depressão para três distritos da cidade do Rio de Janeiro.

\section{Material e Método}

\section{Delineamento}

Os dados apresentados no presente trabalho referem-se ao estudo sobre a população idosa que vem sendo realizado na cidade do Rio de Janeiro ${ }^{37}$. Nesta pesquisa há um sobprojeto sobre saúde mental dos idosos. Este artigo tem como meta apresentar e discutir estes resultados, em particular os aspectos relativos ao estudo de prevalência de Síndrome Cerebral Orgânica - Demência - e Depressão, além de discutir os aspectos metodológicos envolvidos no estudo de confiabilidade e de validação do segmento de saúde mental do questionário utilizado no projeto, denominado BOAS $^{35}$.*

Para tal objetivo, foram realizadas entrevistas em dois momentos. Inicialmente, um trabalhador de campo aplicou o questionário BOAS completo, e, num segundo momento, este entrevistador retornou à residência do entrevistado e repetiu apenas o segmento de saúde mental do questionário, juntamente com um psiquiatra. Em seguida, nesta segunda visita, este psiquiatra avaliou o entrevistado usando a versão para a língua portuguesa do checklist da DSM-III ${ }^{6}$. Deste, foram aplicados os critérios de Síndrome Cerebral Orgânica, e de Depressão. Maior e Distimia para o confronto com os resultados do BOAS $^{39}$.Portanto, realizou-se um estudo de confiabilidade envolvendo duas estratégias (teste-reteste e concordância interavaliador) e um estudo de validação. A Figura 1 resume essa estratégia.

\footnotetext{
* Brazil Old Age Schedule - BOAS 3 é um questionário multidimensional de avaliação funcional desenvolvido para a população idosa de um centro urbano (Rio de Janeiro), baseado em outros instrumentos que obtiveram padroes aceitáveis de validade e confiabilidade, como o questionário $\mathrm{CARE}^{17}$, para o segmento de saúde mental, e o OARS $^{15}$ e PAHO 34 .

O questionário multidimensional visa a cobrir as áreas consideradas mais importantes da vida do idoso, a saber: saúde física, saúde mental, condição social, condição econômica e atividade do dia-a-dia.
}

$\Delta \mathrm{t}$

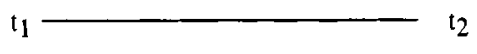

\begin{tabular}{|c|c|c|}
\hline $\begin{array}{l}\text { Entrevista } 1 \text { pelo } \\
\text { entrevistador A, usando } \\
\text { O BOAS completo }\end{array}$ & \multirow[t]{4}{*}{$\begin{array}{l}\text { confiabilida- } \\
\text { de teste } \\
\text { reteste }\end{array}$} & $\begin{array}{l}\text { Entrevista } 2 \text { pelo } \\
\text { entrevistador A, usando } \\
\text { apenas o segmento de } \\
\text { saúde mental do BOAS }\end{array}$ \\
\hline & & $\begin{array}{l}\text { T confiabi- } \\
\text { lidade } \\
\text { intera- } \\
\text { I valiador } \\
\end{array}$ \\
\hline & & $\begin{array}{c}\text { Psiquiatria, } \\
\text { entrevistador } B \text {, obser- } \\
\text { va e marca, usando o } \\
\text { segmento de saúde men- } \\
\text { tal do BOAS }\end{array}$ \\
\hline & & $\begin{array}{c}7 \\
1 \\
1 \\
1 \\
1 \\
\end{array}$ \\
\hline & validaçāo & $\begin{array}{l}\text { Avaliação clínica } \\
\text { do psiquiatra usando } \\
\text { o check-list do } \\
\text { DSM-III }\end{array}$ \\
\hline
\end{tabular}

Figura 1. Delineamento para estudos de confiabilidade e validação: método utilizado no estudo piloto do segmento de saúde mental do questionário BOAS.

\section{Instrumentos}

$\mathrm{O}$ instrumento BOAS tem um segmento de saúde mental que é uma tradução para o português do Short-CARE. A versão do "Short-CARE" utilizada foi a do grupo do "Guy's Hospital and Age Concern Psychogeriatric Research Project," uma adaptação, com pequenas alterações, da versão original do instrumento desenvolvido por Gurland $^{18}$. O processo de tradução do inglês para o português do Short-CARE foi realizado em três tempos ${ }^{38}$. O questionário possui as escalas para avaliação da Síndrome Cerebral Orgânica e Depressão. Para a primeira, há 12 itens que atribuem escores que variam de 0 a 9 pontos, sendo que não foi feita estratificação quanto ao nível de gravidade do quadro. Para a escala de Depressão existem 31 itens, e os escores variam de 0 a 28 pontos.

Os psiquiatras, no estudo de validação, utilizam-se dos critérios do check-list do DSM-III', na versão para a língua portuguesa ${ }^{32}$.

\section{Amostra}

O estudo original foi realizado em três distritos do Rio de Janeiro: Copacabana, Méier e Santa Cruz. Os critérios para seleção dessas áreas são apresentados em outro artigo ${ }^{40}$.

Os estudos de confiabilidade e validade do segmento de Saúde Mental de BOAS foram de- 
senvolvidos em Copacabana. Esta opção decorreu do fato de ser esta região a mais adequada para estudos comparativos com os centros que já utilizaram o "Short-CARE". Nesta área, foram aplicados 252 questionários, e adotaram-se, nesta fase, os pontos de corte utilizados por Lindesay e col. ${ }^{24}$ para definição de casos suspeitos: 2/3 para Síndrome Cerebral Orgânica e $7 / 8$ para Depressão. A partir dos pontos de cortes estabelecidos, 15 idosos foram classificados como portadores de Síndrome Cerebral Orgânica e 58 de Depressão, sendo que destes últimos 7 também apresentaram escores para Síndrome Cerebral Orgânica.

O método de seleção dos entrevistados para os estudos de confiabilidade (teste-reteste e concordância interavaliador) e de validação foi admitir todos os 15 idosos que na primeira entrevista tiveram escores de suspeição de casos para Síndrome Cerebral Orgânica. Para Depressão, dos 58 casos foram retirados os 7 com suspeição dupla de casos, pois já estavam incluídos no grupo de Síndrome Cerebral Orgânica, restando portanto $51 \mathrm{com}$ apenas Depressão. Destes foram aleatoriamente selecionados 15 , mesmo número dos com Síndrome Cerebral Orgânica. Para o grupo controle, dos 179 que apresentaram escores inferiores para ambas as síndromes, foram aleatoriamente selecionados 30 não casos. Em síntese, para a segunda fase de entrevistas o projeto estabeleceu a amostra de 60 idosos. Destes, 30 com suspeição de caso (Síndrome Cerebral Orgânica ou Depressão) e 30 para o controle.

Tendo em vista as características dá investigação que fez uso de um questionário complexo e de um longo tempo de aplicação ${ }^{38}$, o intervalo entre a primeira entrevista e o contacto com o grupo de idosos selecionados para a realização da segunda etapa das entrevistas, demorou, em alguns casos, 9 meses. Este fato fez com que a segunda entrevista, pelo fato de haver alguns idosos com Demência e/ou Depressão, fosse bem mais difícil.

Os critérios de perdas e recusas estabelecidos na primeira fase foram novamente aplicados nesta etapa do estudo. As únicas possibilidades que permitiam substituição eram: morte, doença grave ou mudança de endereço. As recusas só eram admitidas após três tentativas: a primeira ao entrevistador, a segunda ao supervisor de campo e a terceira quando feita ao coordenador do projeto. Caso persistisse a negativa após as três tentativas, este idoso era considerado recusa e não era substituído.

Dos 15 selecionados com suspeição de Síndrome Cerebral Orgânica, um faleceu, não podendo ser substituído pelo fato de não haver sobra para este grupo. Dos 14 restantes, 9 entrevis- tas foram realizadas, e houve 5 recusas. Das 15 entrevistas dos casos de Depressão, 12 foram realizadas, e houve 3 recusas. Dos 7 idosos com suspeição dupla, já incluídos no grupo de Síndrome Cerebral Orgânica, 4 realizaram a entrevista, 2 se recusaram, e o caso de morte acima mencionado ocorreu neste grupo. Dos 30 controles, foram feitas 27 entrevistas, e houve 3 recusas. Neste grupo ocorreram 2 substituições, uma devido à doença e outro por motivo de mudança.

Portanto, foram realizadas 48 entrevistas das 60 previstas, 9 com idosos com suspeição de Síndrome Cerebral Orgânica e 12 com Depressão, sendo que a este grupo se acrescem mais 4 idosos, pela inclusão daqueles com suspeição de dupla patologia (Síndrome Cerebral Orgânica e Depressão), já incluídos no grupo de Síndrome Cerebral Orgânica. A Figura 2 ilustra este procedimento.

\section{Análise}

Estudos de validaçâo - Foram estabelecidos todos os pontos de corte possíveis, sendo calculadas a sensibilidade e a especificidade relativas a cada um desses pontos. Apesar da amostra ser de pequeno tamanho, foram elaborados gráficos com os valores de sensibilidade e proporção de falsos-positivos (Roc-Analysis) ${ }^{31}$ como instrumento auxiliar na escolha dos pontos de corte.

Para validação da escala referente à Síndrome Cerebral Orgânica, comparou-se o escore obtido através do BOAS, por ocasião da segunda entrevista, com a avaliação psiquiátrica no mesmo dia. No entanto, a escala referente ao diagnóstico de Depressão levou em consideração o fato de o segmento de saúde mental do BOAS ter sido positivo na primeira ou segunda entrevista. Tal decisão, cuja implicação será discutida mais adiante, decorreu do fato de os critérios presentes na entrevista psiquiátrica através do checklist do DSM-III levantarem a presença deste transtorno mental em qualquer momento da vida do indivíduo, enquanto o BOAS se restringe apenas ao último mês.

Estudos de confiabilidade - Para medir a concordância interavaliador e teste-reteste entre os escores obtidos na aplicação do segmento de saúde mental do BOAS, foram calculados os índices Kappa, seus intervalos de confiança e testes de significância (para Kappa $=0$ ). Esse procedimento foi implementado pelo programa CONCORD.*

\footnotetext{
* Este programa foi desenvolvido por Klein e Coutinho ${ }^{23}$, e, além dos índices Kappa, gera os diagramas de Bangdiwala ${ }^{7}$ que proporcionam uma representação gráfica da concordância alcançada.
} 


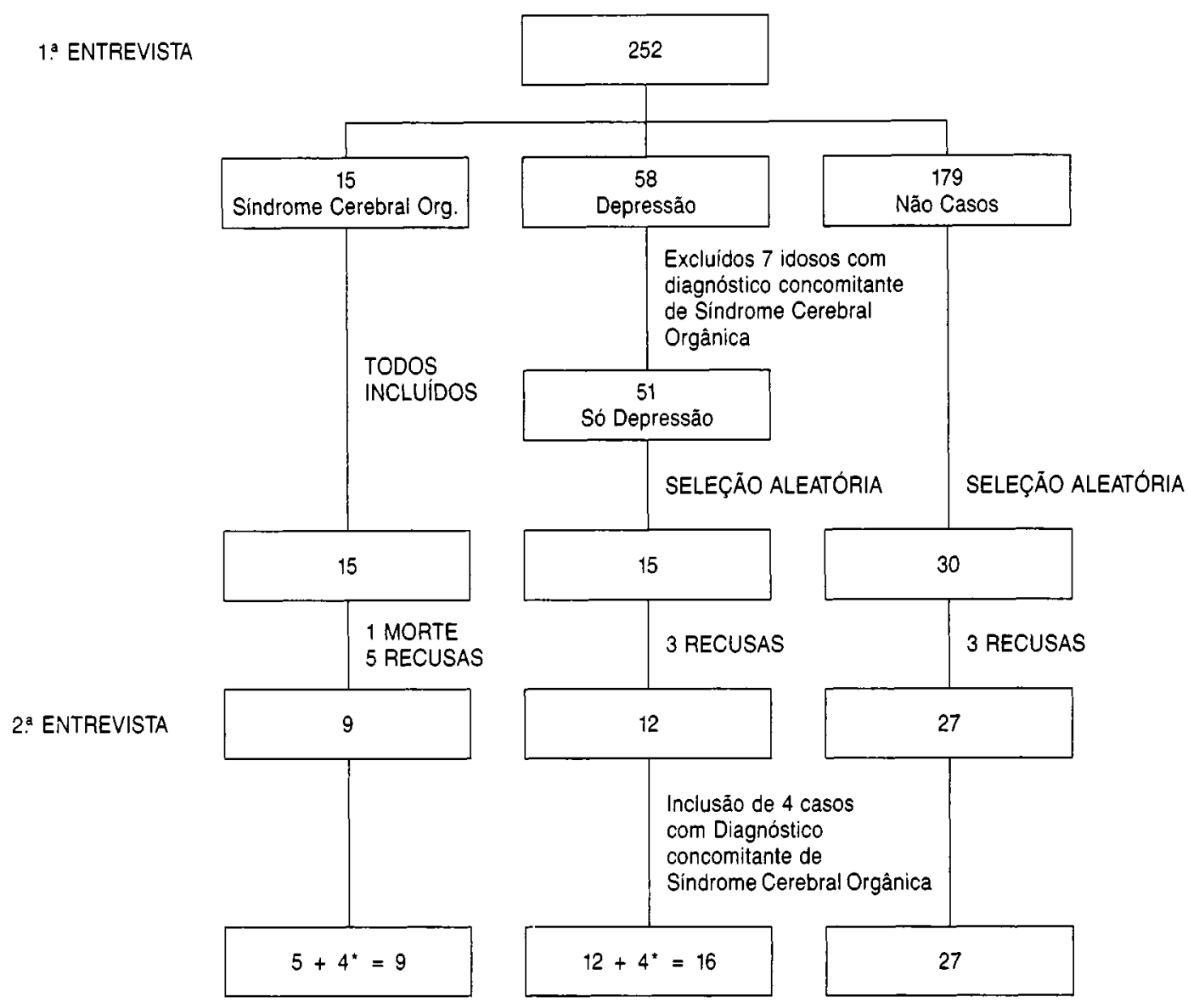

* Os 4 idosos assinalados foram diagnosticados pelo BOAS como Síndrome Cerebral Orgânica e Depressão

Figura 2. Procedimento utilizado na seleção da subamostra para o estudo de validação do questionário BOAS no distrito de Copacabana

\section{Resultados}

\section{Validação}

Para definição dos pontos de corte do segmento de saúde mental de BOAS, levaram-se em consideração os valores obtidos para sensibilidade e especificidade para as várias opções e, conseqüentemente, a proporção de falsos-positivos e falsos-negativos. A Tabela 1 apresenta os valores de sensibilidade e especificidade de 2 pontos

Tabela 1. Valores para sensibilidade e especificidade para os pontos de corte 2/3 e 3/4 para Síndrome Cerebral Orgânica (SCO) e 7/8 e 8/9 para Depressão.

\begin{tabular}{|c|c|c|c|c|}
\hline \multirow{3}{*}{$\begin{array}{l}\text { Valores } \\
\text { Pontos de Corte } \\
\text { Sensibilidade } \\
\text { Especificidade }\end{array}$} & \multicolumn{4}{|c|}{ Doenças } \\
\hline & \multicolumn{2}{|c|}{ SCO } & \multicolumn{2}{|c|}{ Depressão } \\
\hline & $\begin{array}{c}2 / 3 \\
1,00 \\
0,97\end{array}$ & $\begin{array}{c}3 / 4 \\
1,00 \\
1,00\end{array}$ & $\begin{array}{c}7 / 8 \\
1,00 \\
0.79\end{array}$ & $\begin{array}{r}8 / 9 \\
0,89 \\
0,88\end{array}$ \\
\hline
\end{tabular}

de corte para Síndrome Cerebral Orgânica e 2 pontos para Depressão, que mostraram a menor proporção de erros de classificação.

Ao se fazer o gráfico com valores de sensibilidade no eixo y e proporção de falsos-positivos no eixo $x$, os pontos de corte $3 / 4$ para Síndrome Cerebral Orgânica e $8 / 9$ para Depressão foram os que melhor se enquadraram nos critérios no Roc-analysis, ou seja, aquele no qual o ponto se situa mais próximo ao ângulo superior esquerdo do gráfico na relação sensibilidade versus proporção de falsos-positivos ${ }^{36}$.

\section{Confiabilidade}

Assim como no caso da validação, calculouse a concordância interavaliador e teste-reteste com 2 pontos de corte para Síndrome Cerebral Orgânica e 2 pontos de corte para Depressão. Os resultados dos índices Kappa estão na Tabela 2. 
Tabela 2. Valores de Kappa para Sindrome Cerebral Orgânica - SCO (pontos de corte $2 / 3$ e 3/4) e para Depressão (pontos de corte $7 / 8$ e $8 / 9$ ) na confiabilidade teste, reteste e interavaliador.

\begin{tabular}{lcccc}
\hline Valores & \multicolumn{3}{c}{ SCO } & \multicolumn{3}{c}{ Doenças } \\
Depressão \\
\hline Ponto de Corte & $2 / 3$ & $3 / 4$ & $7 / 8$ & $8 / 9$ \\
\hline $\begin{array}{l}\text { Confiabilidade } \\
\text { Teste-Reteste }\end{array}$ & 0,9 & 0,8 & 0,4 & 0,3 \\
$\begin{array}{l}\text { Confiabilidade } \\
\text { Interavaliador }\end{array}$ & 1,0 & 1,0 & 0,8 & 0,3 \\
\hline
\end{tabular}

Pode-se observar que a confiabilidade é elevada para Síndrome Cerebral Orgânica, mas não para Depressão. Nesta, a concordância é baixa quando são comparadas as duas entrevistas em momentos diferentes (confiabilidade/teste-reteste) e entre o trabalhador de campo e o psiquiátra na segunda entrevista (confiabilidade interavaliador) para o ponto de corte $8 / 9$. No caso de diagnóstico de Depressão, apenas a confiabilidade interavaliador no ponto de corte $7 / 8$ foi boa.

\section{Ponto de corte}

Com base nos dados de sensibilidade, especificidade, "Roc-Analysis" e confiabilidade, optou-se por adotar os pontos de corte $2 / 3$ para a escala de Síndrome Cerebral Orgânica e 7/8 para a escala de Depressão.

No caso do diagnóstico de Síndrome Cerebral Orgânica esta decisão ocorreu pelo fato de não ser possível rejeitar a ausência de diferença entre os valores de sensibilidade, especificidade e índice Kappa (para $p<0,05$ ) obtidos com os pontos de corte $2 / 3$ e $3 / 4$. Pelo fato de serem semelhantes, e por ter sido também ligeiramente superior no conjunto das análises realizadas, optou-se um por adotar o primeiro, conforme sugerido no trabalho de Lindesay e col. ${ }^{24}$.

Quando ao diagnóstico de Depressão, observou-se um melhor posicionamento do ponto de corte $8 / 9$ no gráfico de Roc-Analysis, enquanto o ponto de corte $7 / 8$ teve $16,27 \%$ dos indivíduos classificados erroneamente (falsos-positivos ou falsos-negativos) contra $18,6 \%$ do ponto de corte 8/9. Assim como no caso de Síndrome Cerebral Orgânica, não foi possível rejeitar a hipótese nula de que os 2 pontos de corte não diferiam com relação a estes valores. No entanto, face à melhor confiabilidade do ponto de corte $7 / 8$, e também por ter sido o ponto de corte utilizado por Lindesay e col. ${ }^{24}$, optamos por este valor.

\section{Taxas de prevalência}

Na tabela 3 são apresentadas, por distrito, as taxas de prevalência para Síndrome Cerebral Orgânica e Depressão*. Embora tenhamos adotado os pontos de corte $2 / 3$ e $7 / 8$, optamos por apresentar as taxas de prevalência para os pontos $3 / 4$ e $8 / 9$, os quais, em linhas gerais, não se mostram significativamente diferentes, conforme foi discutido antes devido ao tamanho da amostra para os estudos de validação e confiabilidade.

Tabela 3. Taxas de Prevalência de Sindrome Cerebral Orgânica (SCO) e Depressão por distrito de acordo com diferentes pontos de corte (intervalo de confiança de $95 \%$ entre parênteses).

\begin{tabular}{|c|c|c|c|c|}
\hline \multirow{3}{*}{$\frac{\text { Distrito }}{\text { Distrito }}$} & \multicolumn{4}{|c|}{ Doenças } \\
\hline & \multicolumn{2}{|c|}{$\mathrm{SCO}$} & \multicolumn{2}{|c|}{ Depressão } \\
\hline & $2 / 3$ & $3 / 4$ & $7 / 8$ & $8 / 9$ \\
\hline $\begin{array}{l}\text { Copacabana } \\
(n=252)\end{array}$ & $\begin{array}{c}5,9 \\
(2,9)\end{array}$ & $\begin{array}{c}3,9 \\
(2,4)\end{array}$ & $\begin{array}{l}23,0 \\
(5,2)\end{array}$ & $\begin{array}{l}17,5 \\
(4,7)\end{array}$ \\
\hline $\begin{array}{l}\text { Méier } \\
(n=244)\end{array}$ & $\begin{array}{c}9,8 \\
(3,7)\end{array}$ & $\begin{array}{c}6,6 \\
(3,1)\end{array}$ & $\begin{array}{c}20,9 \\
(5,1)\end{array}$ & $\begin{array}{l}17,2 \\
(4,7)\end{array}$ \\
\hline $\begin{array}{l}\text { Santa Cruz } \\
(n=242)\end{array}$ & $\begin{array}{l}29,8 \\
(5,8)\end{array}$ & $\begin{array}{l}19,8 \\
(5,0)\end{array}$ & $\begin{array}{c}36,8 \\
(6,1)\end{array}$ & $\begin{array}{l}28,1 \\
(5,7)\end{array}$ \\
\hline
\end{tabular}

Independentemente dos pontos de corte escolhidos, observa-se uma elevação nas taxas de prevalência para Síndrome Cerebral Orgânica quando se passa do distrito de Copacabana para o Méier e daí para Santa Cruz. Pelo fato de o BOAS e o check-list do DSM-III não distinguirem os diferentes graus de severidade de Síndrome Cerebral Orgânica, as taxas de prevalência foram calculadas globalmente, isto é, para a presença de Síndrome Cerebral Orgânica, sem considerar a gravidade do quadro. Com relação à Depressão, as taxas de Copacabana e Méier situaram-se muito próximas, e o distrito de Santa Cruz apresentou os valores mais elevados.

Para saber se as diferenças entre os três distritos eram decorrentes de uma constituição diferenciada dessas populações, quanto ao sexo e faixa etária, estratificou-se a população de cada região e calcularam-se as taxas de prevalência de Síndrome Cerebral Orgânica e Depressão para homens e mulheres separadamente. Após esse procedimento em relação aos achados anteriores (Tabela 4), quanto à distribuição por sexo, não houve mudança importante. Em relação às faixas etárias, os idosos jovens ( $60 \mathrm{a} 69$ anos) são mais expressivos em Santa Cruz do que nos dois outros distritos.

\footnotetext{
* No censo de 1980, eram idênticas as áreas que comprendiam os 24 distritos definidos pelo IBGE e as 24 Regiōes Administrativas (RAs) estabelecidas pelo Municipio do Rio de Janeiro. Atualmente, a cidade do Rio de Janeiro possui 30 RAs. Possivelmente, no censo deste ano, o IBGE irá trabalhar com 30 distritos.
} 
Tabela 4. Distribuição de idosos por faixa etária e sexo em três distritos do Rio de Janeiro.

\begin{tabular}{|c|c|c|c|c|c|}
\hline \multirow{3}{*}{ Distrito } & \multicolumn{5}{|c|}{ Faixa etária* } \\
\hline & \multicolumn{2}{|c|}{$60-69$} & \multicolumn{2}{|c|}{70 ou + } & \multirow[t]{2}{*}{ Total } \\
\hline & $M$ & $F$ & M & $F$ & \\
\hline Copacabana & $\begin{array}{c}33 \\
(4,5)\end{array}$ & $\begin{array}{c}79 \\
(10,7)\end{array}$ & $\begin{array}{c}59 \\
(8,0)\end{array}$ & $\begin{array}{c}81 \\
(11,0)\end{array}$ & $\begin{array}{c}252 \\
(34,1)\end{array}$ \\
\hline Meiér & $\begin{array}{c}46 \\
(6,2)\end{array}$ & $\begin{array}{c}69 \\
(9,4)\end{array}$ & $\begin{array}{c}50 \\
(6,8)\end{array}$ & $\begin{array}{c}79 \\
(10,7)\end{array}$ & $\begin{array}{c}244 \\
(33,1)\end{array}$ \\
\hline Santa Cruz & $\begin{array}{c}56 \\
(7,6)\end{array}$ & $\begin{array}{c}78 \\
(10,6)\end{array}$ & $\begin{array}{c}40 \\
(5,4)\end{array}$ & $\begin{array}{c}68 \\
(9,2)\end{array}$ & $\begin{array}{c}242 \\
(32,8)\end{array}$ \\
\hline Total & $\begin{array}{c}135 \\
(18,3)\end{array}$ & $\begin{array}{c}226 \\
(30,6)\end{array}$ & $\begin{array}{c}149 \\
(20,2)\end{array}$ & $\begin{array}{c}228 \\
(30,9)\end{array}$ & $\begin{array}{c}738 \\
(100,0)\end{array}$ \\
\hline
\end{tabular}

(1) Percentual

$\mathrm{Na}$ Tabela 5 são apresentadas as taxas de prevalência corrigidas* pelos valores de sensibilidade e especificidade obtidos com os pontos de corte $2 / 3$ para Síndrome Cerebral Orgânica e 7/8 para Depressão. O padrão observado entre os diferentes distritos não foi modificado significativamente após a correção das taxas de prevalência, sobretudo se considerarmos que os intervalos de confiança destas estimativas são muito grandes.

Tabela 5. Taxas de prevalência corrigidas de Sindrome Cerebral Orgânica (SCO) e Depressāo, para os pontos de corte $2 / 3$ e $7 / 8$, por distrito.

\begin{tabular}{lrc}
\hline Distrito & SCo & Doença \\
\hline $\begin{array}{l}\text { Copacabana } \\
(n=252)\end{array}$ & 2,6 & 3,1 \\
$\begin{array}{l}\text { Méier } \\
(n=244)\end{array}$ & 6,6 & 0,4 \\
$\begin{array}{l}\text { Santa Cruz } \\
(n=242)\end{array}$ & 27,2 & 20,4 \\
\hline
\end{tabular}

\section{Comentários}

Antes de analisarmos as taxas de prevalência de Síndrome Cerebral Orgânica e Depressão encontradas no presente estudo, gostaríamos de abordar alguns aspectos que envolvem a validação e o estudo de confiabilidade do instrumento utilizado para os diagnósticos. Os valores de sensibilidade e especificidade aqui apresentados de forma pontual envolvem intervalos de confiança grandes em função da pequena subamostra utilizada para este fim. Esse aspecto não tem sido discutido nos estudos de validação de instrumentos de diagnóstico, apesar de as amostras

\footnotetext{
$\underset{\substack{\text { Prevalência } \\ \text { corrigida }}}{\text { Prevalência BOAS }) \cdot(\text { Falso-positivo })}(1)$
}

freqüentemente não serem maiores do que a desta investigação. Este fato reveste-se de grande importância nas decisões sobre os pontos de corte a serem adotados, assim como no cálculo das prevalências corrigidas a partir das prevalências encontradas com o instrumento da investigação, no nosso caso o BOAS, e dos valores de sensibilidade e de especificidade. Estes, por serem variáveis aleatórias, estão também sujeitos a flutuações cuja magnitude depende da sua variância e do tamanho da amostra.

Em nosso caso, esses aspectos se manifestaram através de mudanças bruscas na sensibilidade e especificidade obtidas quando mudamos de um ponto de corte para outro vizinho. Assim, a escolha desses pontos de corte deve ser feita com alguma reserva por não se poder afirmar, com elevado grau de confiança, que as proporções de falsos-positivos e falsos-negativos sejam diferentes para pontos de corte limítrofes.

Outra questão a ser discutida, ainda com relação ao estudo de validação dos diagnósticos formulados a partir do BOAS, foi a decisão de considerar suspeitos de Depressão os idosos que tenham alcançado escores superiores ao ponto de corte estabelecido (7/8), na primeira ou na segunda entrevista. Assim, os idosos que foram positivos pelo BOAS na primeira entrevista, mas não na segunda (ocasião em que o psiquiatra esteve presente para a avaliação), foram considerados casos. Tal decisão decorreu do fato de o BOAS levar em consideração apenas os 30 últimos dias, enquanto o check-list do DSM-III investiga para qualquer momento da vida do entrevistado.

Ainda com relação a essa questão, chamou atenção o fato de haver um padrão na confiabilidade teste-reteste do BOAS, quando houve escore elevado para Depressão em uma ocasião (entrevista) e não em outra. Em praticamente todos esses casos discordantes $(88,9 \%)$ o escore foi superior ao ponto de corte na primeira entrevista e inferior na segunda. Se apenas o intervalo de tempo entre as duas entrevistas fosse o motivo para a mudança num quadro que está restrito a apenas 30 dias, esperaríamos encontrar uma proporção de idosos cujo BOAS foi positivo na primeira entrevista e negativo na segunda, semelhante a de idosos cujo BOAS foi negativo na primeira entrevista e positivo na segunda.

Portanto, supomos que, além do problema do enfoque diferenciado do tempo entre o BOAS e o check-list, deva haver algum outro aspecto que tenha contribuído para uma possível negação de fatores que conduziram ao diagnóstico de suspeição de Depressão pelo BOAS na segunda entrevista. Uma hipótese seria a me- 
nor disponibilidade pessoal do entrevistado para colaborar pela segunda vez, e, pela negação sistemática, ele a encerraria mais rapidamente. Este fato deve ser considerado quando se utiliza questionários de longo tempo de aplicação e se solicita ao entrevistado permissão para um possivel retorno.*

Quanto à confiabilidade interavaliador, esta foi muito baixa $(\mathrm{Kappa}=0,29)$ quando se comparou suspeitos e não-suspeitos de Depressão pelo BOAS, com ponto de corte 8/9. Entretanto, como a grande maioria dessas discordâncias envolveu valores limítrofes, ao se trabalhar com o ponto de corte de $7 / 8$ a confiabilidade elevouse para 0,78 .

Uma última questão a ser abordada quanto ao diagnóstico de Depressão foi a decisão de se utilizar os critérios diagnósticos de Depressão maior e distimia do check-list do DSM-III para o procedimento de validação da escala de Depressão do BOAS. Esta decisão foi discutida em outro artigo ${ }^{39}$ e deveu-se, essencialmente, à observação, durante o estudo piloto, de que os itens presentes no BOAS relacionavam-se com os critérios de ambas as categorias diagnósticas.

Todas essas considerações relativas ao diagnóstico de Depressão não se aplicam, em nossa opinião, ao diagnóstico de Síndrome Cerebral Orgânica pelo fato de as perguntas referentes a essa categoria nosológica serem de caráter mais objetivo e, principalmente, porque o quadro clínico de demência tem um caráter crônico e progressivo, não sendo esperadas mudanças para melhor, da primeira para a segunda entrevista. No caso de confiabilidade interavaliador, os valores Kappa encontrados foram altos para ambas as entrevistas.

Tendo sido feitas estas considerações, alguns aspectos das taxas de prevalência obtidas no estudo merecem ser abordados. As estimativas descritas para Síndrome Cerebral Orgânica e Depressão, em particular para a primeira, têm se mostrado muito diferentes de uma investigação para outra. Em nosso estudo, as taxas para Síndrome Cerebral Orgânica situaram-se ao longo de um grande espectro, sendo mais baixas nos distritos de Copacabana $(5,9 \%)$ e Méier $(9,8 \%)$ e muito elevadas em Santa Cruz $(29,8 \%)$. A provável explicação para os baixos valores de Copacabana e Méier pode ser atribuída à faixa etária de idosos do estudo, onde quase a metade é de idosos jovens (60 a 69 anos) (Tabela 4). Em relação a Santa Cruz, a explicação visivelmente é outra, já que nesse distrito o núme-

\footnotetext{
* A média do tempo de aplicação do BOAS, nos 48 idosos, por ocasião da primeira entrevista, foi consideravelmente maior do que na segunda.
}

ro de idosos da faixa etária de 60 a 69 anos é muito maior do que nos outros dois distritos e, portanto, se deveria esperar um menor número de idosos com Síndrome Cerebral Orgânica em relação aos dois outros distritos estudados. Atribuímos o problema à inadequação do questionário ao universo cultural e social desta população idosa $^{16}$. Algumas perguntas não são compatíveis com o baixo nível de escolaridade verificado neste distrito, conforme pode ser observado na Tabela 6 .

Tabela 6. Comparação entre níveis de escolaridade dos idosos nos distritos selecionados, através do teste binomial para proporçōes.

\begin{tabular}{lllll}
\hline $\begin{array}{l}\text { Escolaridade } \\
\text { Cruzamento }\end{array}$ & Nenhuma & Primária & $\begin{array}{l}\text { Nivel } \\
\text { Médio }\end{array}$ & $\begin{array}{l}\text { Nivel } \\
\text { Superior }\end{array}$ \\
\hline Copacabana & -6.737 & -9.316 & 6.138 & $29.275(1)$ \\
$x$ & $p<0,001$ & $p<0,001$ & $p<0,001$ & $p<0,001(2)$ \\
Méier & rejeita a & $\begin{array}{l}\text { rejeita a } \\
\text { igualdade }\end{array}$ & $\begin{array}{l}\text { rejeita a } \\
\text { igualdade }\end{array}$ & $\begin{array}{l}\text { rejeita a (3) } \\
\text { igualdade }\end{array}$ \\
\hline Copacabana & -9.537 & -11.923 & 16.821 & 38.638 \\
$x$ & $p<0,001$ & $p<0,001$ & $p<0,001$ & $p<0,001$ \\
Santa Cruz & rejeita a & rejeita a & rejeita a & rejeita a \\
& igualdade & igualdade & igualdade & igualdade \\
\hline Méier & -3.738 & -2.577 & 8.344 & 1.142 \\
$x$ & $p<0,001$ & $p=0,005$ & $p<0,001$ & $p=0,125$ \\
Santa Cruz & rejeita a & rejeita a & $\begin{array}{l}\text { rejeita a } \\
\text { najo rejeita }\end{array}$ \\
& igualdade & igualdade & igualdade & igualdade \\
\hline
\end{tabular}

Nota: (1) Valor da estatistica do teste.

(2) p-valor.

(3) Decisão do teste a nivel de significância de $5 \%$.

No caso do diagnóstico de Depressão, embora o distrito de Santa Cruz tenha apresentado as taxas mais elevadas $(36,8 \%)$, Copacabana e Méier não diferiam (23,0 e 20,9\%, respectivamente). Embora nesse caso as diferenças entre os distritos não tenham sido tão grandes quanto para o diagnóstico de Síndrome Cerebral Orgânica, chamou a atenção a magnitude dessas taxas de prevalência, sobretudo em Santa Cruz, se compararmos com os dados da literatura.

É possível que aspectos relacionados com a confiabilidade e a validação do BOAS tenham contribuído para esta discrepância. Conforme assinalamos anteriormente, foram identificados problemas quanto ao período de tempo avaliado pelo BOAS ( 30 dias) e pelo check-list da DSM-III (toda a vida). Esse fato nos levou a optar por considerar como casos suspeitos de depressão os indivíduos que tiverem escores superiores a 7 pontos na primeira ou na segunda entrevista. Durante a discussão dessa estratégia, observamos uma concentração de escores positivos para depressão na primeira entrevista em relação a segunda entrevista (confiabilidade testereteste), que pode indicar a presença de um viés 
na avaliação desta categoria nosológica em nossa amosta. Esses fatores, associados a possíveis problemas de adequação do instrumento ao universo cultural e social da população investiga$\mathrm{da}$, podem ter contribuído para que as taxas de prevalência de depressão em nosso estudo fossem mais elevadas do que o encontrado na literatura.

Veras, R.P. \& Coutinho, E. da S.F. [Prevalence of Depression and Organic Cerebral Syndrome in the elderly population (Brazil)] Rev. Saúde públ., S. Paulo, 25: 209-17, 1991. Data from a prevalence study of Organic Cerebral Syndrome and Depression in an elderly population living in three boroughs of Rio de Janeiro city are presented. The methodological issues related to interrater and test-retest reliability are discussed and the cut-off point for the instrument adapted (BOAS) established. The prevalence rates in the three boroughs were found, respectively, to be: $5.9 \%, 9.8 \%$ and $29.8 \%$ for Organic Cerebral Syndrome and 20.9\%, 23.0\% and $36.8 \%$ for Depression. The prevalence rats have been adjusted using information on sensitivity and specificity for both diagnoses. Aspects of these differences are discussed in the light of national and international literature.

Keywords: Aged. Depression, epidemiology. Organic mental disorders, epidemiology. Epidemiologic methods.

\section{Referências Bibliográficas}

1. ALMEIDA FILHO, N. Epidemiologia sem números: uma introdução crítica à ciência epidemiológica. Rio de Janeiro, Campus, 1989.

2. ALMEIDA FILHO, N. \& BASTOS, S. Estudo caso-controle de associação e desordens depressivas em mulheres. J.bras. Psiquiat., 31:25-30, 1982.

3. ALMEIDA FILHO, N. \& SANTANA, V.S. Espaço urbano e doença mental: um estudo de área ecológica. Cad. Saúde públ., 2:334-48, 1986.

4. ALMEIDA FILHO, N. et al. Estudo epidemiológico dos transtornos mentais em uma população de idosos. área urbana de Salvador - BA. J. bras. Psiquiat., 33: 114-20, 1984.

5. ALMEIDA FILHO, $N$. et al. Relações entre a saúde mental dos pais e a saúde mental das crianças em uma população urbana de Salvador - Bahia. Acta Psiquiat. Amer. Lat., 31:211-21, 1985.

6. AMERICAN PSYCHIATRIC ASSOCIATION. Committee on Nomenclature and Statistics. Diagnostic and statistical manual of mental disorders. $3^{\text {rd }} \mathrm{ed}$. Washington, D.C., 1980.

7. BANGDIWALA, S.I. A graphical test for observer agreement. [Apresentado ao ISI Statistical Meeting, Amsterdan, 1985-mimeografado].

8. BLAY, S. Instrumentos de avaliação em epidemiologia psicogeriátrica. Rev. $A B P-A P A L, 11$ (2):45-54, 1989.

9. BLAY, S. Psychogeriatric activity in Brazil. Int. Psychogeriat., 1:107-11, 1989.

-10. BLAY, S. Revisão e crítica da metodologia dos estudos sobre epidemiologia dos distúrbios psiquiátricos na população idosa. Rev. $A B P-A P A L, 11$ (1):45-54, 1989.

11. BLAY, S. et. al. Validity on Brazilian version of the Older American Resources Services (OARS) mental health screening questionnaire. J. Amer. Geriat. Soc. , 36: 687-92, 1988.
12. COUTINHO, E.S.F. Confiabilidade do diagnóstico psiquiátrico em hospitais do Rio de Janeiro. Rio de Janeiro, 1987. [Dissertação de Mestrado . Escola Nacional de Saúde Pública - FIOCRUZ].

13. COUTINHO, E.S.F. et al. Confiabilidade do diagnóstico psiquiátrico em hospitais do Rio de Janeiro. J. bras. Psiquiat., 37: 197-200, 1988.

14. COUTINHO, E.S.F. \& KLEIN. C.M, Fontes de discordância do diagnóstico psiquiátrico. Bol. Ofic. sanit. panamer., 106:390-406, 1989.

15. DUKE UNIVERSITY OARS. Multidimentional functional assessment: the OARS methodology. $2^{\text {nd }}$ ed. Durham, N.C., Duke University. Centre for the Study of Ageing and Human Development, 1978.

16. GURLAND, B.J. The boderland of demencia: the influence of sociocultural characteristics and rates of dementia occuring in the senium. In: Miller, N.E. \& Cohen, G.D., eds. Clinica: aspects of Alzheimer's disease and senile dementia: Aging. New York, Raven Press, 1981. v. 15.

17. GURLAND, B. et al. The comprehensive assessment and referral evaluation (care): rationale, development and reliability. Int. J. Aging hum. Develop., 8: 9-42, $1977 / 78$.

18. GURLAND, B. et al. The short-care: an efficient instrument for the assessment of depression, dementia and disability. J. Geront., 39:166-9, 1984.

19. IACOPONI, E. et al. Ensino de psicologia médica em um ambulatório de medicina geral e familiar. Bol. Psiquiat., 17: 130-5, 1984.

20. KANEKO, Z. Epidemiological studies on mental disorders of the aged in Japan. In: Proceedings of $8^{\text {th }}$, Abstracts of simposia and lectures. Int. Assoc. Gerontol., 1969. p. $284-7$.

21. KAY, D.W.K. \& GRAHAM, D.B. The epidemiology of mental disorders in the aged: handbook of studies on psychiatry and old age. Oxford, Elservier Science Publ., 1984.

22. KAY, D.W.K. et al. Old age disorders in newcastleupon-tyne: a study of prevalence. Brit. J. Psychiat., 110:146-58, 1964

23. KLEIN, C.M. \& COUTINHO, E.S.F. Confiabilidade do diagnóstico psiquiátrico: um programa prático para microcomputadores. J. bras. Psiquiat., 37:51-4, 1988.

24. LINDESAY, J. BRIGGS, K.; MURPHY, E. The Guy's Age Concern Survey: prevalence rates of cognitive impairment, depression and ansiety in a urban elderly community. Brit. J. Psychiat., 155: 317-29, 1989.

25. MARI, J. Psychiatric morbidity in three primary medical care clinics in the city of São Paulo. Soc. Psychiat., 22:129-38, 1987.

26. MARI, J, \& WILLIAMS, P. A comparison of the validity of two psychiatric screening questionnaires (GHQ, 12 and SRQ-20) in Brazil, using relative operating characteristics (roc) analysis. Psychol. Med. 15:651-9, 1985.

27. MARI, J. \& WILLIAMS, P. A validity study of a psychiatry screening questionnaire (SRQ-20) in primary care in the city of São Paulo. Brit. $J$. Psychiat., 148: 23-6, 1986.

28. MARI, J. \& WILlIAMS, P. Misclassification by psychiatric screening questionnaires. J. chron. Dis., 30:371-8, 1986.

29. MARI, J. et al. Confiabilidade da versão brasileira da entrevista para estudos comunitários. Bol. Ofic. sanit. panamer., 100: 77-82, 1986.

30. MARI, J. et al. Detection of psychiatric morbidity in the primary medical care setting in Brazil. Rev. Saúde públ., S. Paulo, 21:501-7, 1987. 
31. MERTZ, C.E. et al. Rocifit. Chicago, Department of Radiology and the Franklin McLean Memorial Research Institute. University of Chicago, 1984.

32. MIRANDA, C. et al. Diagnostic Interview Schedule (DIS) version. III: adaptação para o Brasil da versão portuguesa original feita pelo Departamento de Saúde Mental da Faculdade de Medicina do Porto. São Paulo, Departamento de Psiquiatria da Escola Paulista de Medicina, 1985. [Mimeografado].

33. MORGADO, A.F. \& COUTINHO, E.S.F. Dados de epidemiologia descritiva de transtornos mentais em grupos populacionais do Brasil. Cad. Saúde públ., 3:327-47, 1985.

34. PAN-AMERICAN HEALTH ORGANIZATION. Epidemiology of ageing in Latin America and Caribean. Washington, D.C., sd. [Mimeografado].

35. QUESTIONNAIRE BOAS. Brazil Old Age Schedule (Multidimensional questionnaire for the elderly population). Rio de Janeiro, Instituto de Medicina Social da UERJ, 1987.

36. SWETS, J.A. Roc-analysis applied to the evaluation of medical imaging techniques. Invest. Radiol., 14(2): 109-21, 1979.

37. VERAS, R. A multidimensional assessment of the elderly population living in Rio de Janeiro. London,
Guy's Hospital Medical School, London University, 1986. [Protocolo de Projeto de Doutorado].

38. VERAS, R. \& COUTINHO, E.S.F. População idosa no Rio de Janeiro (Brasil): estudo - piloto da confiabilidade e validação do segmento de saúde mental do questionário BOAS. Rev. Saúde públ., S. Paulo, 24:156-63, 1990.

39. VERAS, R. et al. Pesquisando populaçס̃es idosas - a importância do instrumento $\mathrm{e}$ do treinamento de equipe: uma contribuição metodológica. Rev. Saúde públ., S. Paulo, 22:513-8, 1988.

40. VERAS, R. et al. Proposta metodológica para inquérito domiciliar com populações idosas na cidade do Rio de Janeiro (Brasil). Rev. Saúde públ., S. Paulo, 23: 429-38, 1989.

41. WORLD HEALTH ORGANIZATION. Scientific Group on Dementia in Late Life: Research and Action, Paris, 1983. Report. Geneva, 1986. (Technical Report Series, 730).

Recebido para publicação em $23 / 10 / 1990$ Reapresentado em 1/3/1991 Aprovado para publicação em 8/3/1991 